EPJ Web of Conferences 52, 07004 (2013)

DOI: 10.1051/epjconf/20135207004

(C) Owned by the authors, published by EDP Sciences, 2013

\title{
Proton-air and proton-proton cross sections
}

\author{
Ralf Ulrich ${ }^{1, a}$ \\ ${ }^{1}$ Karlsruhe Institute of Technology, Germany
}

\begin{abstract}
Different attempts to measure hadronic cross sections with cosmic ray data are reviewed. The major results are compared to each other and the differences in the corresponding analyses are discussed. Besides some important differences, it is crucial to see that all analyses are based on the same fundamental relation of longitudinal air shower development to the observed fluctuation of experimental observables. Furthermore, the relation of the measured proton-air to the more fundamental proton-proton cross section is discussed. The current global picture combines hadronic proton-proton cross section data from accelerator and cosmic ray measurements and indicates a good consistency with predictions of models up to the highest energies.
\end{abstract}

\section{Introduction}

The energy of cosmic ray particles extends far beyond the reach of current accelerator experiments. Already since the 1960ies this has been exploited to evaluate hadronic proton-air cross sections at energies above the respective reach in energy of accelerators at any given point in time. Before the first precise measurements made at the ISR became available (e.g. [1]), it was cosmic ray data that unambiguously indicated the presence of the pomeron and the begin of a rise of the hadronic cross sections towards higher energies [2]. And very recently it was argued that cosmic ray data may indicate that the black disc limit in proton-proton collisions has already been reached [3].

In general we find that the global picture of high energy accelerator measurements of proton-proton cross sections, the extrapolation of those to higher energies by interaction models and cosmic ray measurements of protonair cross sections show in general no significant disagreement [4].

In section 2 the different experimental analysis approaches to measure the proton-air cross section are related to a simplified model of longitudinal air shower development and in section 3 the existing data are reviewed. The conversion of proton-air to proton-proton cross sections and the comparison of cosmic ray to accelerator data is shown in section 4. How precise data of air shower fluctuations can be used directly to constrain ultra-high energy cross sections is the topic of section 5 .

\section{Longitudinal development of extensive air showers}

At energies above $\sim 10^{15} \mathrm{eV}$ cosmic ray particles can only be detected indirectly via the observation of extensive air

\footnotetext{
a e-mail: ralf.ulrich@kit.edu
}

shower cascades in the atmosphere. The interaction length of ultra-high energy particles in the atmosphere is one of the key parameters that defines the properties of these cascades. This has the consequence that all interpretations of air shower observations are subject to uncertainties due to a limited knowledge of these interactions. However, this can be exploited since it means that air shower observations are sensitive to features of hadronic interactions and in particular to ultra-high energy hadronic cross sections.

In Fig. 1 a very reduced model of longitudinal air shower development is shown. There are three distinct stages of a cosmic ray induced air shower in the atmosphere: (i) the region up to $X_{1}$ where the primary particle did not yet inelastically collide with the atmosphere, (ii) the range between the first inelastic collision, which marks the starting point of the actual air shower cascade, and the atmospheric slant depth where the shower cascade reaches its maximum extend $X_{\max }$ and (iii) the development after the shower maximum, which is characterized by the absorption of the cascade in the atmosphere.

The average depth of the first interaction, $\left\langle X_{1}\right\rangle=\lambda_{\mathrm{p} \text {-air }}$, and thus the starting of the air shower cascade, is directly related to the inelastic cross section via $\sigma_{\text {p-air }}=$ $\left\langle m_{\text {air }}\right\rangle / \lambda_{\mathrm{p}-\text { air }}$, where $\left\langle m_{\text {air }}\right\rangle$ is the mean target mass of air and $\lambda_{\mathrm{p}-\text { air }}$ is identical to $\left\langle X_{1}\right\rangle$ due to the exponential distribution of $X_{1}$. At ultra-high energies $X_{1}$ cannot be measured directly. On the other hand, the depth of the shower maximum is a typical air shower observable, which can be measured with good precision (c.f. [5]). In order to determine the cross section from measurements of the air shower maximum, $X_{\max }$, it is necessary to correct for the additional air shower cascade within the slant depth range $\Delta X_{1}$ [6]. This can be done only with Monte Carlo simulations. Thus, depending on the implementation of the data analysis there is some level of uncertainty related to the modelling of extensive air showers and hadronic interactions at ultra-high energies in particular. 


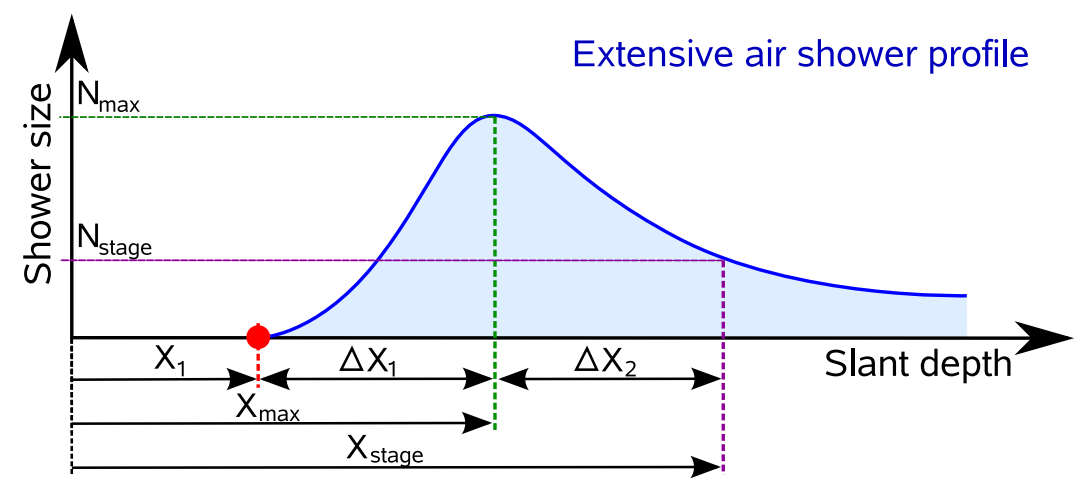

Figure 1. Stages of longitudinal air shower development. The slant depth range from the first interaction to the shower maximum is called $\Delta X_{1}$ and the range from the shower maximum to the observation ground level is $\Delta X_{2}$. From [6].
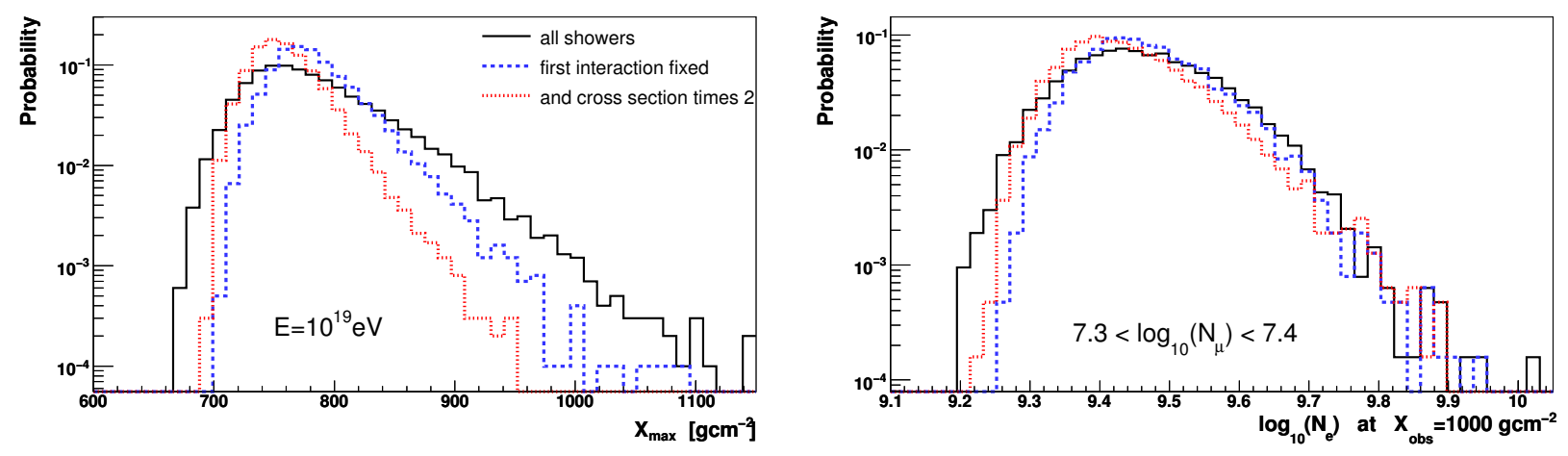

Figure 2. Shower-to-shower fluctuations of telescope (left) and ground array (right) for primary protons. The solid line shows the prediction of the original QGSJETII model [8], while the dashed line is for showers having their first interaction at the fixed slant depth corresponding to the predicted proton-air interaction length of QGSJETII. The dotted line is for simulated air showers with increased cross section by a factor of two, $f_{19}=2$, in addition to the fixed first interaction point.

In Fig. 1 it is also visible how measurements of the longitudinal air shower development at a fixed atmospheric depth $X_{\text {stage }}$ are sensitive to $X_{1}$. For cosmic ray showers of the same primary energy the shower size at $X_{\text {stage }}$ depends on the distance to $X_{\max }$ and thus to $X_{1}$. The larger distance to $X_{1}$ reduces the sensitivity of such ground based measurements to the cross section with respect to the direct observation of $X_{\max }$ with telescopes (c.f. Fig. 2). In addition, also the air shower development occurring in the range of $\Delta X_{2}$ must be corrected for with Monte Carlo simulations, which introduces further model dependence.

With telescope based observations of the longitudinal air shower cascade the energy of the primary particle can be estimated from the integral of the observed energy deposit in the atmosphere. The depth of the shower maximum is a direct observable of telescopes. With air shower arrays the experimental situation is very different from that. The primary energy of a particular air shower event needs to be estimated from the shower size at ground level [9-11]. For this purpose the total signal in the muon component of the air shower cascade is best suited, but cannot be measured by all experiments in the same way. Also in general it requires a conversion technique relying on the modeling of the extensive air shower cascade with Monte Carlo simulations. Given a possibility to estimate the primary energy, the electromagnetic shower size at ground level, thus the signal in electrons, positrons and photons, can be used to determine the stage of attenuation of the air shower cascade in the atmosphere. This is an indirect measure of the depth distance to the shower maximum $X_{\max }$ and is then also sensitive to $X_{1}$. The concrete implementation of how this is achieved by the analyses of different Collaborations differ from each other, mostly because of the differing experimental situations and observables that are available.

All measurements of the cross section, and thus $X_{1}$, are based on the observation of the longitudinal air shower development. The relation between air shower array and telescope observations is visualized in Fig. 1.

\section{Existing cosmic ray measurements}

In Fig. 3 the cosmic ray based measurements of the protonair cross section are compared to model predictions. The results at lower energies [13-15] are from the direct observation of cosmic ray protons in the atmosphere, the other data are from air shower measurements. All air shower 


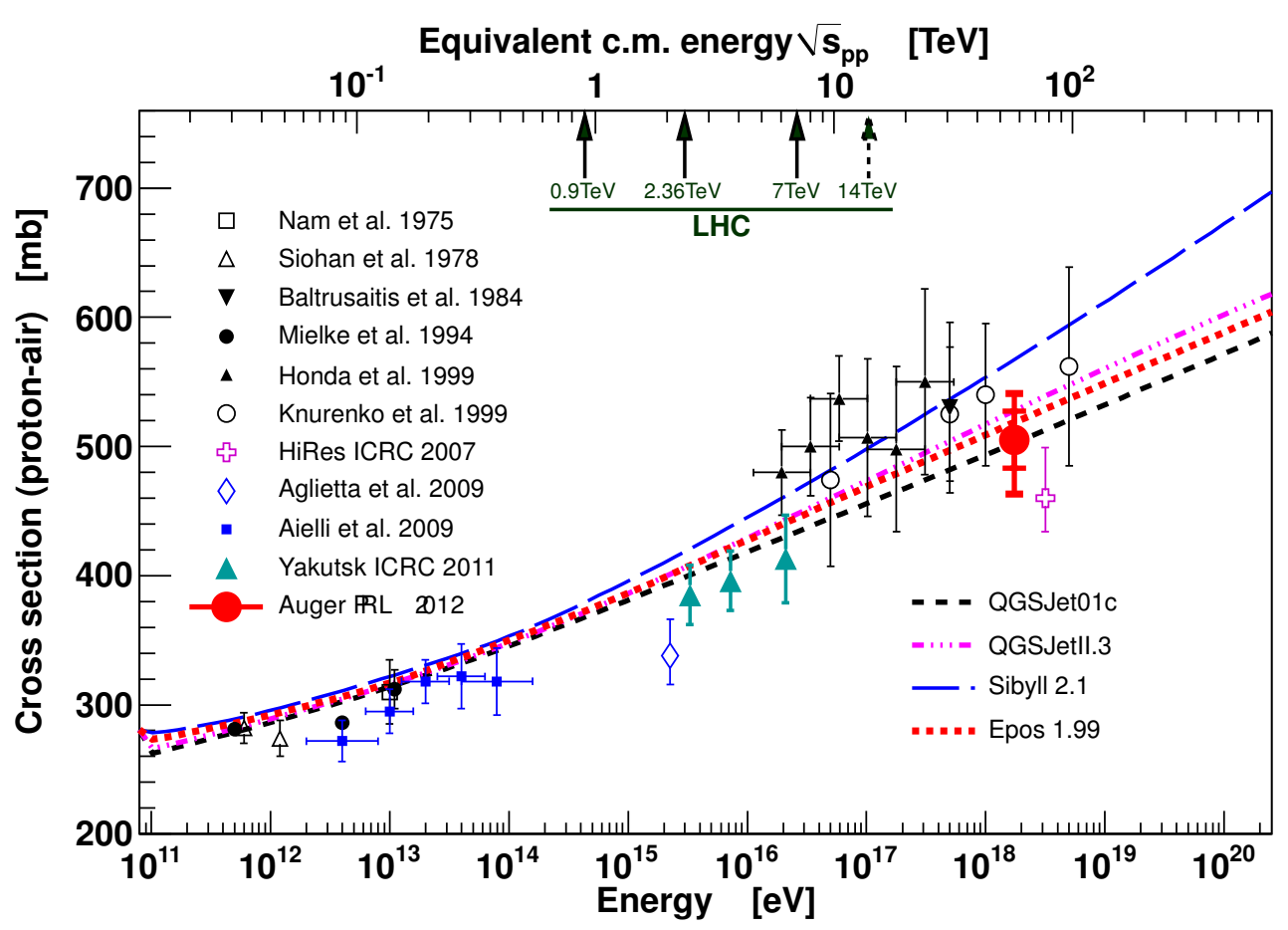

Figure 3. Compilation of cosmic ray proton-air cross section measurements. The predictions of hadronic interaction models are also shown. The results at lower energies are mostly from the observation of unaccompanied hadrons, while at higher energies are all from air shower based analyses. Plot from [12].

Table 1. Overview of the air shower based analyses of the proton-air cross section. The energy range of the analysis is quoted, as well as the number of events used for the analysis $N_{\text {evt }}$, the experimental resolution of $X_{\max }$, the observed exponential attenuation of air showers in the atmosphere, and the derived proton-air cross section with statistical uncertainties.

\begin{tabular}{l|ccccc} 
Experiment & $\lg E / \mathrm{eV}$ & $N_{\mathrm{evt}}$ & $\sigma_{\text {res }}\left(X_{\max }\right) / \mathrm{gcm}^{-2}$ & $\Lambda_{\text {obs }} / \mathrm{gcm}^{-2}$ & $\sigma(\mathrm{p}-\mathrm{air}) / \mathrm{mb}$ \\
\hline \hline Fly's Eye & 17.7 & $\approx 500$ & 70 & $73 . \pm 9$ & $530 \pm 66$ \\
AGASA & $16.2-17.6$ & 553065 & $\mathrm{n} / \mathrm{a}$ & $\mathrm{n} / \mathrm{a}$ & $480-550$ \\
HiRes & 18.5 & 1348 & $\approx 27$ & $63.2 \pm 4.7$ & $460 \pm 49$ \\
EAS-TOP & 15.3 & $\mathrm{O}\left(10^{7}\right)$ & $\mathrm{n} / \mathrm{a}$ & $\mathrm{n} / \mathrm{a}$ & $338 \pm 41$ \\
ARGO-YBJ & $12.6-13.9$ & $\mathrm{O}\left(10^{8}\right)$ & $\mathrm{n} / \mathrm{a}$ & $\mathrm{n} / \mathrm{a}$ & $272-318$ \\
Yakutsk $^{*}$ & $16.5-18.5$ & 1783 & $80-90$ & $\mathrm{n} / \mathrm{a}$ & $470-550$ \\
Auger & 18.24 & 3082 & $<25$ & $55.8 \pm 2.8$ & $505 \pm 23$
\end{tabular}

based results are based on the measurement of the attenuation of air showers in the atmosphere. The HiRes approach is slightly different, since it determines the properties of the distribution of $X_{1}$ by unfolding the $X_{\max }$-distribution by using a $\Delta X_{1}$-distribution obtained from air shower simulations [16]. However, at the same time HiRes also explicitly measures and quotes the attenuation of the air showers in the atmosphere.

The experimental data of all these analyses are summarized in Tab. 1 . The quantity $\Lambda_{\mathrm{obs}}$ is the observed attenuation coefficient of the air shower cascades in the atmosphere. Depending on the experimental resolution, it can be more or less affected by experimental effects and these results are not corrected for this. Tails in the experimental resolution will make the observed attenuation coefficient larger.

To derive the proton-air cross section from measurements of $\Lambda_{\text {obs }}$ a relation of the form $\Lambda_{\text {obs }}=k \lambda_{\text {int }}$ is used, where the factor $k$ takes into account all experimental effects as well as the impact of the air shower process within the slant depth interval $\Delta X_{1}$. To understand this better it is useful to distinguish these different parts as $k=k_{\text {det }} k_{\text {eas }}$, and it was argued that the primary effect responsible for $k_{\text {eas }} \neq 1$ is the inelasticity of interactions, yielding $k_{\text {eas }}=1 /\left(1-\left\langle\left(1-k_{\text {in }}\right)^{\gamma-1}\right\rangle\right)$. Furthermore, it was shown that $k_{\text {eas }}$ itself depends on the cross section at ultra-high energies, since in the very beginning of the air shower cascade, interactions at energies only slightly below the initial primary energy $E_{0}$ dominate the air shower properties. This latter effect is taken into account only by the analysis of the Pierre Auger Collaboration by using effectively a parameterization $k=k\left(\sigma_{\mathrm{p} \text {-air }}\right.$, det $)$. The effect of the detector resolution in the analysis is implicitly taken into account by all analyses by the use of realistic detector simulations. The ARGO Collaboration explicitly quotes $k_{\operatorname{det}}=1.15$ to 1.45 , and the Yakutsk 
Collaboration $k_{\text {det }}>1$ [17]. Because of $k_{\text {det }} \neq 1$ it is not possible to compare the experimental results of $\Lambda_{\text {obs }}$ directly to each other, furthermore, it is not possible to use a common value of $k$ derived purely from theory for several experiments.

\section{Relation of proton-air to proton-proton cross sections}

The proton-air interaction length is the quantity that directly determines the longitudinal development of extensive air showers. Thus the primary sensitivity of cosmic ray experiments if for the proton-air cross section. In order to derive a more fundamental proton-proton cross section from this, nuclear effects must be corrected for. The most straightforward framework to calculate nuclear effects for this purpose has been proposed by Glauber et al [18]. It takes into account the nuclear geometry and the multiple scattering nature of nuclear collisions. In the original calculation light nuclei are described by the shell model taking into account the most fundamental nucleonnucleon correlations, but other higher-order effects, like for example inelastic screening, are not included. There are several models available to extend the original calculation of Glauber (for example [8, 19-23]), but there is no theory from first principles available and none of the existing models may be considered complete. It is thus important to identify the minimum model that can be applied for the conversion and to find the smallest parameter set necessary. Furthermore, the parameter space must be reduced with the help of the available accelerator data as far as possible. This is a the approach chosen by the Pierre Auger Collaboration.

The Glauber calculation with a shell type description of the nitrogen nuclei is extended with a description of inelastic screening according to Good\&Walker [24]. The screening is tuned to accelerator data of single diffraction in combination with proton-carbon cross section measurements, and it was found that low-mass diffraction with $\xi>0.05$ results in a model that can reproduce all nucleonnucleon as well as proton-carbon cross section that have been measured at accelerators.

The comparison of proton-air and proton-proton cross sections using physical models for the description of nuclear effects are not only providing some insight into hadronic collisions at energies beyond current accelerator measurements, they also test the physics of nuclear collisions used in the conversion calculations. In particular with precise measurements taken at similar center-of-mass energy this can become sensitive to probe the physics of interesting effects, like inelastic screening, and saturation.

\section{Air shower fluctuations as lower limit on cross sections}

One of the primary sources of fluctuations of air showers is the interaction length of cosmic ray primaries in the atmosphere. Modern cosmic ray experiments can measure air shower fluctuations with good precision and small experimental uncertainties [5]. Data from different experiments is summarized in Fig. 6. Also the HiRes experiment published results on air shower fluctuations [25], however, an observable is used, which is defined quite specifically for the HiRes experiment, and also detector effects are not corrected for. Thus, this data cannot be directly compared to other data, and it cannot be used for the following analysis.

The fluctuation of the depth of the shower maximum can be expressed at the sum of fluctuations of the first interaction point plus the fluctuations arising from the subsequent air shower development up to the shower maximum

$$
V\left[X_{\max }\right]=V\left[X_{1}\right]+V\left[\Delta X_{1}\right]
$$

The quantity $V\left[\Delta X_{1}\right]$ can only be studied with air shower Monte Carlo simulations, however, it will always yield: $V\left[\Delta X_{1}\right]>0$. Thus, we arrive at the following inequality

$$
V\left[X_{\max }\right]>V\left[X_{1}\right]
$$

where $V\left[X_{1}\right]=\lambda_{\text {int }}^{2}=\left(\left\langle m_{\text {air }}\right\rangle / \sigma_{\text {int }}\right)^{2}$. This leads to

$$
\sigma_{\text {int }}>\left\langle m_{\text {air }}\right\rangle / \sqrt{V\left[X_{1}\right]},
$$

which means that a measurement of the fluctuations of $X_{\max }$ is putting a lower limit on the cross section. The experimental resolution of a measurement of $X_{\max }$ would add directly to Eq. (1) but this does not change any the conclusions. It only requires a very precise measurement, otherwise the limit from Eq. (2) will not restrict the relevant phase space.

The question about the primary cosmic ray mass composition is more difficult to handle. The limit of Eq. (2) depends on the primary type of the cosmic ray particles. Thus, by default it is a limit on the cosmic ray-air cross section. The mixture of several cosmic ray species leads to non trivial effects on the resulting $V\left[X_{\max }\right]$. For example, with respect to a pure proton composition, up to a certain amount of admixture $V\left[X_{\max }\right]$ will increase before it starts to decrease.

In Fig. 7 the 90\% C.L. limits of the fluctuation data are shown for the data sets with sufficiently precise measurements. The resulting limits on the cross section of the most precise experiment are most constraining $(\rightarrow$ highest limits), which is what is expected from statistics.

\section{Summary}

All cosmic ray based cross section measurements above $10^{15} \mathrm{eV}$ are based on the analysis of air shower data. The observation of air shower and air shower fluctuations in particular can be very sensitive to fluctuations of the starting depth of the air shower cascade. The primary source of all fluctuations is the first interaction of the cosmic ray particle in the atmosphere. The exponential distribution of $X_{1}$ is directly related to the inelastic proton-air cross section, however, this is not a property of extensive air showers that is subject to direct observation. Typical air shower observables are the location of the shower maximum $X_{\max }$, or 


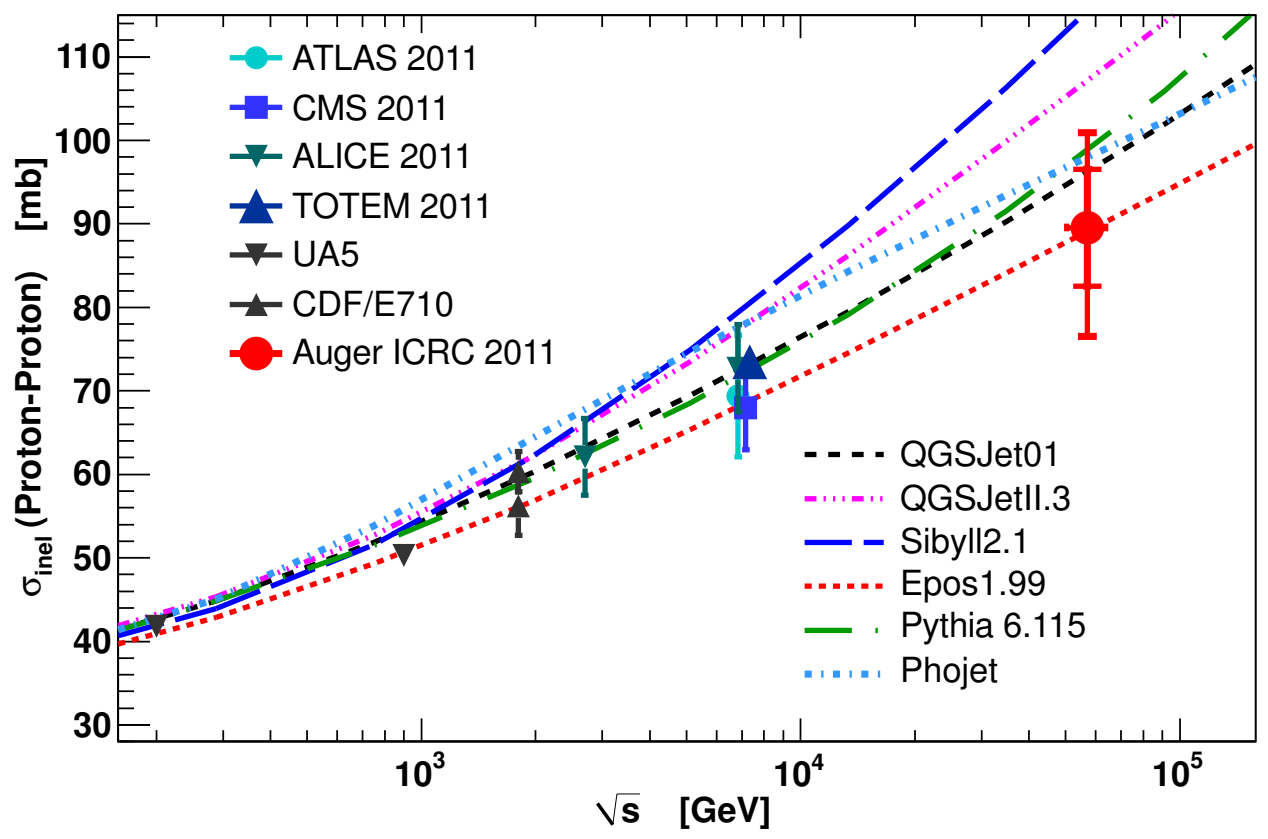

Figure 4. Data on the inelastic hadronic cross section at very high energies. Model predictions are also shown. The LHC and also Pierre Auger data are favoring the models with a moderate rise of the cross section with energy. From [12].

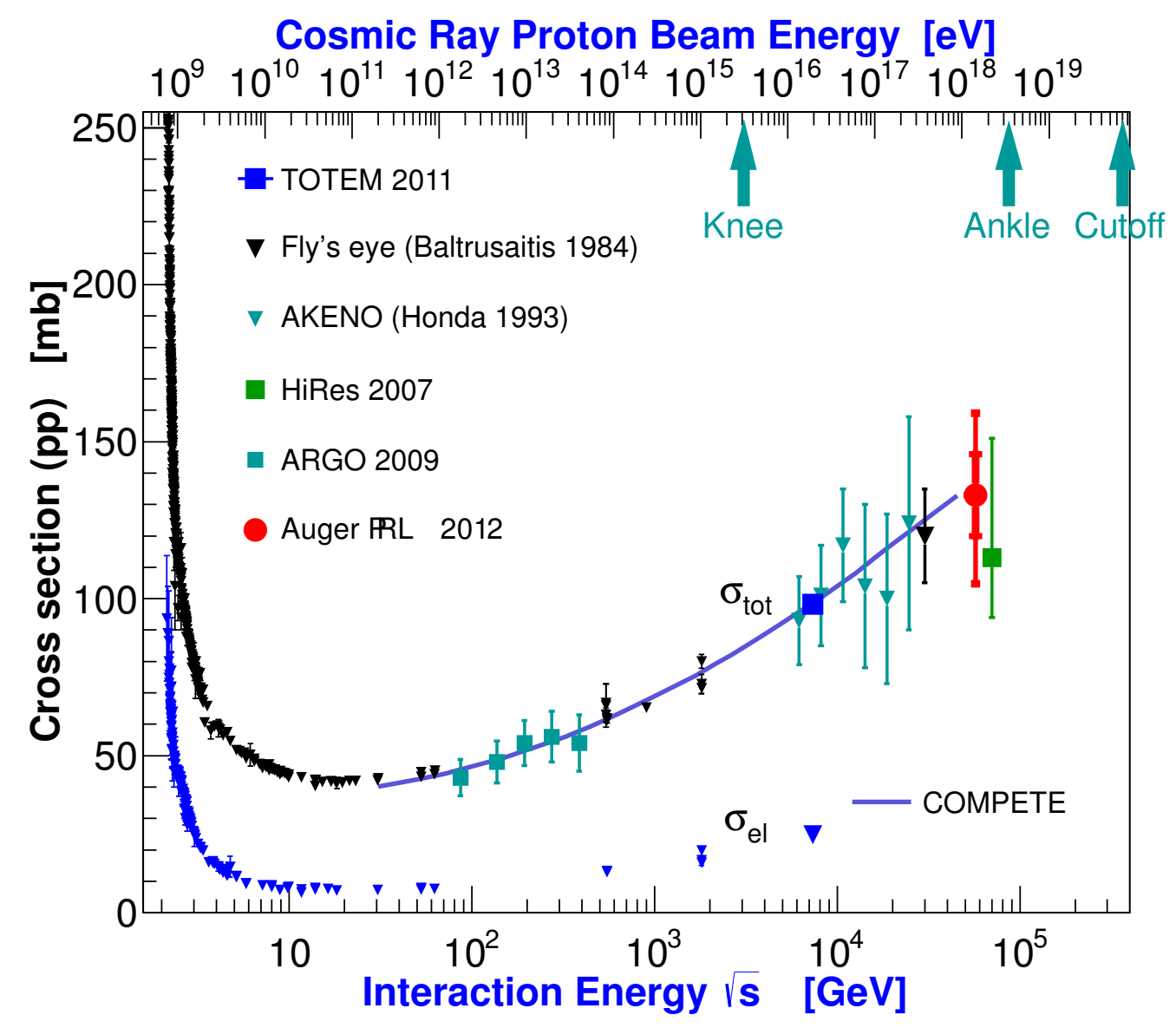

Figure 5. Compilation of data of the total hadronic cross section including accelerator as well as cosmic ray data. The COMPETE fit is the best phenomenological description of these data without using LHC as well as cosmic ray data as a constraint. 


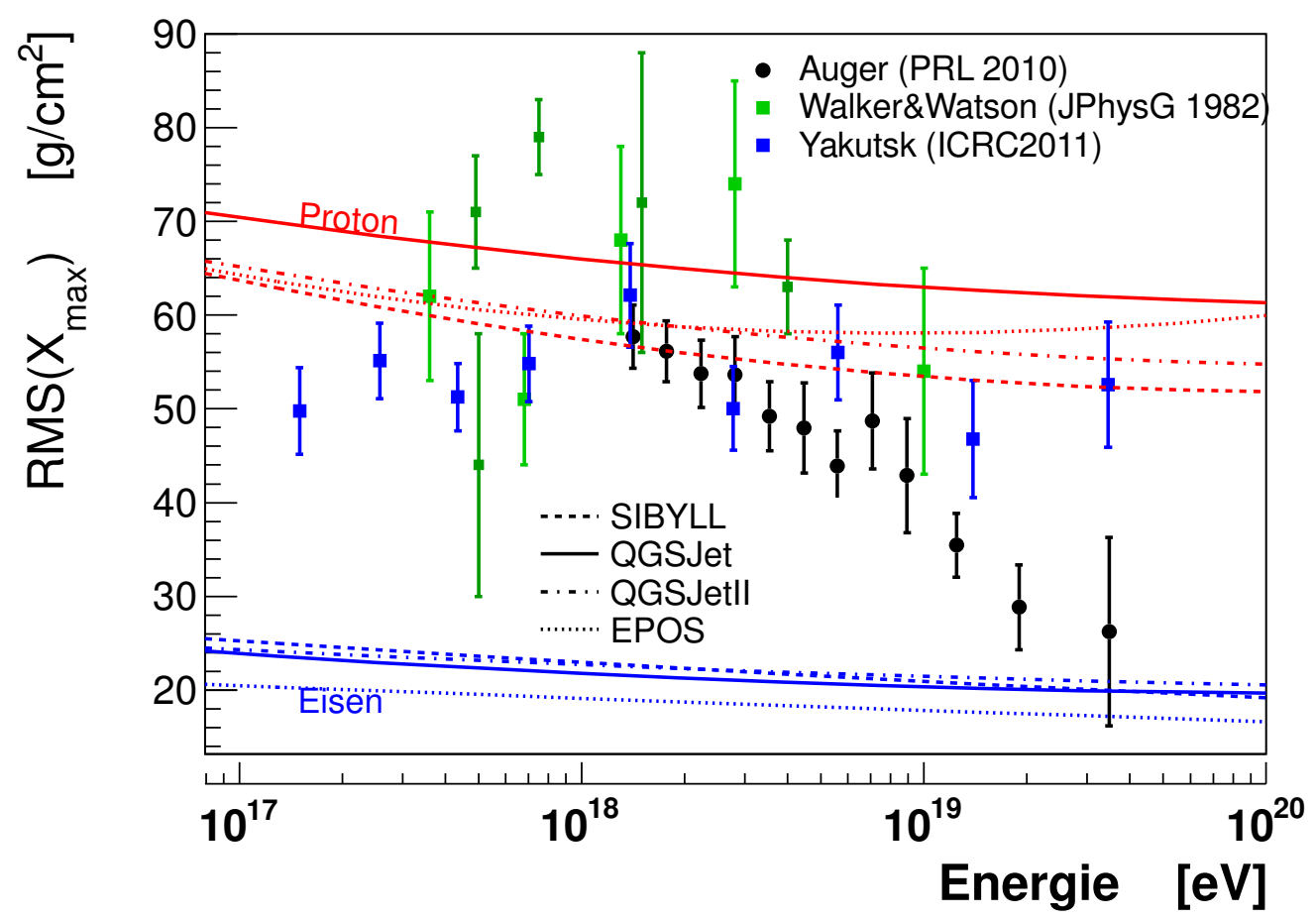

Figure 6. Published fluctuations of $X_{\max }$ together with model predictions for proton and iron induced air showers.

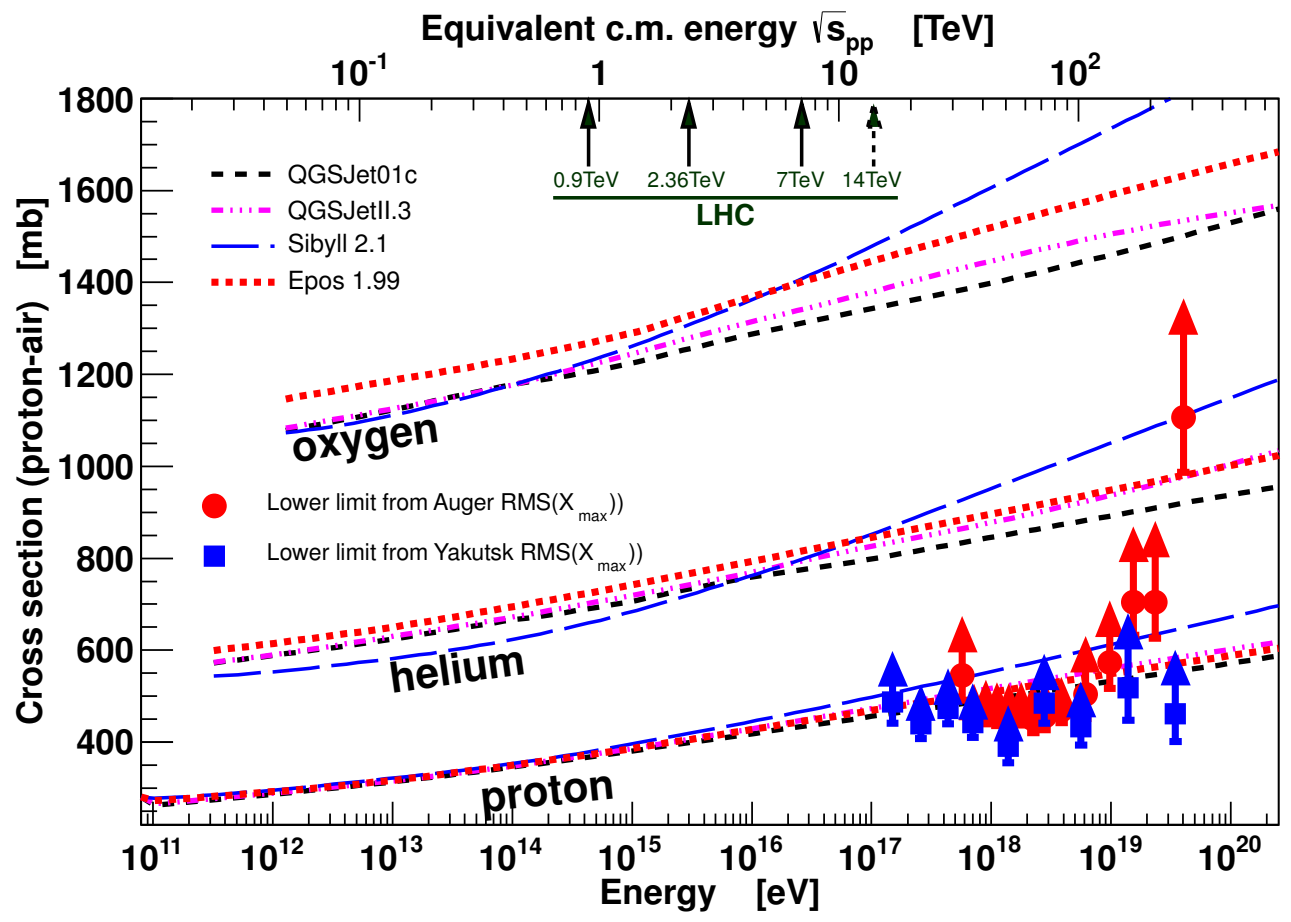

Figure 7. The $90 \%$ C.L. lower limit of the cross section derived directly from the RMS of $X_{\max }$. No detector resolution or air shower fluctuations are corrected for. 
the shower size at observation level measured with an air shower array at ground level. With a simple model of the longitudinal air shower development these very different observables can be related to fluctuations of $X_{1}$ in a common framework. Still, the measurement of $X_{\max }$ is more sensitive to $X_{1}$ than the measurements obtained from air shower arrays, which can be understood from additional model dependent corrections necessary for the later.

The primary result of cosmic ray data analyses is the proton-air cross section, since this is what directly determines the features of the air shower cascade. The relation of the proton-air to the proton-proton cross sections requires additional model assumptions to correct for nuclear effects. These corrections add additional systematic uncertainties to the cosmic ray data. Due to the very high energy of some of the cosmic ray measurements, even with larger uncertainties, the data has still potential for constraining the extrapolation of hadronic interaction models towards ultra-high energies. On the other hand, with more precise cosmic ray measurements undertaken at the energies, where also accelerator data from the LHC will become available, a direct test of the models used to correct for the nuclear effects can be feasible. This will reduce the systematic uncertainties in modelling of extensive air showers, and it will also increase the sensitivity of cosmic ray measurements to fundamental hadronic physics at ultrahigh energies. Effects like saturation or inelastic screening may be directly tested in such comparisons.

But even without any complicated data analysis, precise cosmic ray data can be directly sensitive to hadronic cross sections. With very much improved detection resolution in the current generation of cosmic ray experiments, at ultra-high energies, it became feasible to measure the longitudinal fluctuations of air showers over wide ranges in primary energy. This is a very recent success in cosmic ray research, and it indicates a surprising feature: The fluctuations of air shower cascades seems to decrease after the "ankle" feature in the spectrum of cosmic rays. This is not yet fully understood and one of the most interesting points of current research in cosmic ray physics. One advantage of measuring small fluctuations with good experimental precision is that it can be utilized to constrain the largest sources of fluctuations in air showers, which is the primary proton-air cross section. The data of the Pierre Auger Observatory indicates so small fluctuations at the highest published energy bins that this is incompatible with the assumption of a pure proton model together with a standard extrapolation of the proton-air cross section to these energies. Either, the composition is not dominated by protons at these energies, or the proton-air cross section must start to diverge quite severely from the standard extrapolations at center-of-mass energies around $200 \mathrm{TeV}$.

\section{References}

[1] N. Grigorov et al., Proc. of $9^{\text {th }}$ Int. Cosmic Ray Conf., London 1, 860 (1965).

[2] G. Yodh, S. Tonwar, T. Gaisser and R. Ellsworth, Phys. Rev. D 27, 1183 (1983).

[3] M. Block and F. Halzen, Phys. Rev. D 86, 051504 (2012) [arXiv:1208.4086 [hep-ph]].

[4] M. Menon and P.Silva, arXiv:1212.5096 [hep-ph].

[5] J. Abraham et al., Phys. Rev. Lett. 104, 091101 (2010).

[6] R. Ulrich et al., New J. Phys. 11, 065018 (2009).

[7] R. Ellsworth et al., Phys. Rev. D 26, 336 (1982). R. Baltrusaitis et al., Phys. Rev. Lett. 52, 1380 (1984).

[8] S. Ostapchenko, Phys. Rev. D 81, 114028 (2010).

[9] M. Honda et al., Phys. Rev. Lett. 70, 525 (1993).

[10] M. Aglietta et al., EAS-TOP Collaboration, Phys. Rev. D 79, 032004 (2009).

[11] G. Aielli et al., ARGO Collaboration, Phys. Rev. D 80, 092004 (2009).

[12] P. Abreu et al. [Pierre Auger Collaboration], Phys. Rev. Lett. 109, 062002 (2012) [arXiv:1208.1520 [hepex]].

[13] R. Nam et al., Proc. of $14^{\text {th }}$ Int. Cosmic Ray Conf., Munich 7, 2258 (1975).

[14] F. Siohan et al., J. Phys. G 4, 1169 (1978).

[15] H. Mielke et al., J. Phys. G 20, 637 (1994).

[16] K. Belov et al., Nucl. Phys. Proc. Suppl. 151, 197 (2006).

[17] S. Knurenko et al., Proc. of $26^{\text {th }}$ Int. Cosmic Ray Conf., Salt Lake City 1, 372 (1999).

[18] R. Glauber, Phys. Rev. 100, 242 (1955). R. Glauber and G. Matthiae, Nucl. Phys. B 21, 135 (1970).

[19] N. Kalmykov, and S. Ostapchenko, Phys. Atom. Nucl. 56, 346 (1993).

[20] V. Guzey, M. Strikman, Phys. Lett. B 633, 245 (2006).

[21] G. Baym, B. Blattel, L.L. Frankfurt, H. Heiselberg and M. Strikman, Phys. Rev. C 52, 1604 (1995).

[22] D.R. Harrington, Phys. Rev. C 67, 064904 (2003).

[23] L. Frankfurt, G. Miller, M. Strikman, Phys. Rev. Lett. 71, 2859 (1993).

[24] M. Good and W. Walker, Phys. Rev. 120, 1857 (1960).

[25] R. U. Abbasi et al. [HiRes Collaboration], Phys. Rev. Lett. 104, 161101 (2010) [arXiv:0910.4184 [astroph.HE]]. 